\title{
Robust passive reliable control for uncertain discrete-time Systems with time delay and actuator faults
}

\author{
Chunxia Fan ${ }^{1}$, Feifei Sheng ${ }^{2}$ \\ College of Automation, Nanjing University of Posts and Telecommunications, Nanjing, 210003 \\ E-mail: njfcx@126.com, feifeisheng555@163.com
}

\begin{abstract}
Keywords: uncertain discrete-time system, time delay, passive control, actuator faults, reliable control
\end{abstract}

\begin{abstract}
For a class of uncertain discrete-time system with time delay and actuator faults, a state feedback controller is designed based on passivity control method. The possible failure of each actuator is described by a variable varying within a given interval. Our aim is to design a reliable state feedback controller such that the closed-loop system is robust and strictly passive in the case of parameter uncertain and possible actuator failure. In terms of Lyapunov-Krasovskii functional and linear matrix inequality approach, a sufficient condition is presented for the existence of controller in the form of linear matrix inequalities (LMI). Finally, we give two numerical examples to demonstrate the effectiveness of the proposed design techniques.
\end{abstract}

\section{INTRODUCTION}

In a real engineering, time delays often occur such as chemical engineering, heating process. It is well known that time delay is one of major sources of instability or degradation in performance of a control system. And parameter uncertainties are unavoidable because there some variations in system parameters and modeling errors or some ignored factors in a modeling system. Therefore, increasing attentions have been attracted on some dynamical systems with parameter uncertainties and time delays [1, 2, 3, 4].

Passivity plays an important role in actual control systems, which is a special case of the dissipation [5, 6]. In the description of passivity, it takes the arithmetic product of the input and output of systems as the supply rate. And the supply rate contributes to the storage function. Because the storage function of passive systems can link with the Lyapunov-Krasovskii function of complex systems. Therefore, the method of passive analysis is used to investigate robust stability of systems with time delays $[1-4,7,8]$.

On the other hand, the actuator is the most failed part in a control system because it is always under the circumstance of implanting the mission of controller. Therefore, fault-tolerance strategy of actuator should be taken seriously in a control system. The problem of robust fault-tolerance control is studied for nonlinear stochastic systems [9] and uncertain singular systems [10]. The problem of robust dissipative control for a class of uncertain continuous time system with input delay and linear fractional uncertainties despite possible actuator failures [11]. And in [12], the design method of a robust passive controller is presented for an uncertain stochastic switched time-delay systems with actuator failures. Robust passive fault-tolerance control is not only in theoretical research, but also in experiment research [13, 14]. For energy management of electrical vehicle system, a passive fault-tolerant controller is presented based on $\mathrm{H}$-infinity approach [13]. In order to monitor the Fuel Metering Valve of a jet engine, a virtual sensor is designed based on robust on-line estimation [14].

In the references above mentioned, the fault tolerant control problem is studied by using passivity control method for continuous time systems. However, the discrete-time system can describe many engineering phenomenon due to computation component or communication network introduced into the control engineering. Therefore, the problem of robust passive reliable control is still needed to be investigated deeply for an uncertain discrete-time system with time delay and actuator faults. In this paper, we design a reliable controller to guarantee the performance of an 
uncertain discrete-time system in the presence of time delay as well as actuator failures based on the passive control method.

\section{PROBLEM FORMULATION}

Consider the following uncertain discrete-time system with time delay

$$
\begin{gathered}
x(k+1)=(A+\Delta A) x(k)+\left(A_{1}+\Delta A_{1}\right) x(k-d) \\
+(B+\Delta B) u(k)+D w(k) \\
z(k)=C x(k)+E w(k) \\
x(k)=\varphi(k) \quad k \in[-d, 0]
\end{gathered}
$$

where $x(k) \in R^{n}, u(k) \in R^{m}, z(k) \in R^{p}, w(k) \in R^{q}, \varphi(k)$, represent system state, control input, controlled output, external disturbance, initial state respectively. $w(k) \in l_{2}[0, \infty) ; A, A_{1}, B, C, D, E$ are known and real matrices with proper dimensions. $\Delta A, \Delta A_{1}, \Delta B$ represent the uncertainty of the system structure.

Assumption 1 Uncertainties $\triangle A, \Delta A_{1}, \Delta B$ satisfy

$$
\left[\Delta A \Delta A_{1} \Delta B\right]=H G(k)\left[M_{0} M_{1} M_{2}\right]
$$

where $G^{T}(k) G(k) \leq I ; H, M_{0}, M_{1}, M_{2}$ are known and real matrices with proper dimensions.

When an actuator fault occurs, we use $u^{F}(k)$ to describe the control signal sent from actuators. Here, consider the actuator fault model [15] with failure parameter $F$ :

$$
u^{F}(k)=F u(k)
$$

The failure parameter $F$ satisfies the following

$$
0 \leq \underline{F}=\operatorname{diag}\left\{\underline{f}_{1}, \cdots, \underline{f}_{m}\right\} \leq \operatorname{diag}\left\{f_{1}, \cdots, f_{m}\right\} \leq \bar{F}=\operatorname{diag}\left\{\bar{f}_{1}, \cdots, \bar{f}_{m}\right\} \leq I
$$

where the variables $f_{i}(i=1, \cdots, m)$ quantify the actuator control effectiveness.

Let:

$$
\begin{aligned}
& F_{0}=\operatorname{diag}\left(f_{01}, \cdots, f_{0 m}\right) \triangleq(\underline{F}+\bar{F}) / 2 \triangleq \operatorname{diag}\left(\left(\underline{f_{1}}+\bar{f}_{1}\right) / 2, \cdots,\left(\underline{f}_{m}+\bar{f}_{m}\right) / 2\right) \\
& \widetilde{F}=\operatorname{diag}\left(\widetilde{f}_{1}, \cdots, \widetilde{f}_{m}\right) \triangleq(\bar{F}-\underline{F}) / 2 \triangleq \operatorname{diag}\left(\left(\bar{f}_{1}-\underline{f_{1}}\right) / 2, \cdots,\left(\bar{f}_{m}-\underline{f}_{m}\right) / 2\right)
\end{aligned}
$$

Apparently, $\tilde{f}_{i}=\left(\bar{f}_{i}-\underline{f}_{i}\right) / 2(i=1, \cdots, m)$ and we can rewrite $F$ as follows:

$$
F=F_{0}+\Delta \triangleq F_{0}+\operatorname{diag}\left(\delta_{1}, \cdots, \delta_{m}\right)
$$

where $\left\|\delta_{i}\right\| \leq \tilde{f}_{i}(i=1, \cdots, m)$.

We consider the following controller

$$
u(k)=K x(k)
$$

where $K$ is the controller gain to be designed.

Then, we can obtain the following loop-closed system

$$
\begin{aligned}
& x(k+1)=\bar{A} x(k)+\overline{A_{1}} x(k-d)+D w(k) \\
& z(k)=C x(k)+E w(k)
\end{aligned}
$$

where $\bar{A}=A+\triangle A+B F K+\triangle B F K, \bar{A}_{1}=A_{1}+\Delta A_{1}$.

Definition 1 [16]: The uncertain discrete-time delay system (7) is said to be strictly passive if there exist a positive definite function $V(x(k))$, such that the following holds

$$
\Delta V=V(x(k+1))-V(x(k))<z^{T}(k) w(k)
$$

for any $k \geq 0$ and any $w(k)$.

Lemma 1 [17]: Matrices $X_{1}, X_{2}, X_{3}$ with appropriate dimensions, satisfying the following

$$
X_{1}+\varepsilon^{-1} X_{3} X_{3}^{T}+\varepsilon X_{2}^{T} X_{2}<0, \quad \exists \varepsilon>0
$$

only when $X_{1}+X_{3} \Delta_{k} X_{2}+X_{2}^{T} \Delta_{k}^{T} X_{3}^{T}<0$, where $\quad \forall \Delta_{k} \Delta_{k}^{T} \leq I$. 


\section{MAIN RESULTS}

In this section, a reliable controller is designed to guarantee the system (7) being robust stable and strictly passive.

Theorem 1: Suppose that Assumption 1 holds. If there exist positive definite symmetric matrices $Z$ and $X$, matrix $K_{l}$ and constant $\varepsilon>0$ satisfying the following

$$
M_{1}=\left[\begin{array}{ccccccc}
-Z & * & * & * & * & * & * \\
0 & -X & * & * & * & * & * \\
C Z & 0 & -E^{T} & * & * & * & * \\
A Z+B F K_{l} & A_{1} X & D & -Z+\varepsilon H H^{T} & * & * & * \\
M_{0} Z+M_{2} K_{l} & M_{1} X & 0 & 0 & -\varepsilon I & * & * \\
0 & 0 & 0 & 0 & 0 & -\varepsilon I & * \\
Z & 0 & 0 & 0 & 0 & 0 & -X
\end{array}\right]<0
$$

then system (7) is robust and strictly passive. And the controller gain is given as follows:

$$
K=K_{l} Z^{-1}
$$

Proof : Define the following Lyapunov-Krasovskii functional candidate:

$$
V(x(k))=x^{T}(k) P x(k)+\sum_{\tau=k-d}^{k-1} x^{T}(\tau) Q x(\tau)
$$

then get the difference of (12) along (7)

$$
\begin{aligned}
& \Delta V(k)=V(x(k+1))-V(x(k)) \\
= & x^{T}(k+1) P x(k+1)-x^{T}(k) P x(k)+\sum_{\tau=k-d+1}^{k} x^{T}(\tau) Q x(\tau)-x^{T}(k) P x(k)-\sum_{\tau=k-d}^{k-1} x^{T}(\tau) Q x(\tau) \\
= & {\left[\bar{A} x(k)+\bar{A}_{1} x(k-d)+D w(k)\right]^{T} P *\left[\bar{A} x(k)+\bar{A}_{1} x(k-d)+D w(k)\right] } \\
& -x^{T}(k) P x(k)+x^{T}(k) Q x(k)-x^{T}(k-d) Q x(k-d) \\
= & x^{T}(k)\left(\bar{A}^{T} P \bar{A}-P+Q\right) x(k)+x^{T}(k-d)\left(\bar{A}_{1}^{T} P \bar{A}_{1}-Q\right) x(k-d)+w^{T}(k) D^{T} P D w(k) \\
& +x^{T}(k) \bar{A}^{T} P \bar{A}_{1} x(k-d)+x^{T}(k) \bar{A}^{T} P D w(k)+x^{T}(k-d) \bar{A}_{1}^{T} P \bar{A} x(k) \\
& +x^{T}(k-d) \bar{A}_{1}^{T} P D w(k)+w^{T}(k) D^{T} P \bar{A} x(k)+w^{T}(k) D^{T} P \bar{A}_{1} x(k-d)
\end{aligned}
$$

Let

$$
\xi=\left[\begin{array}{lll}
x^{T}(k) & x^{T}(k-d) \quad w^{T}(k)
\end{array}\right]^{T}, \quad \zeta=\left[\begin{array}{lll}
x^{T}(k) & x^{T}(k-d)
\end{array}\right]^{T}
$$

When $w(k)=0$, it follows from (13) that $\Delta V=\zeta^{T}(k) \Pi_{1} \zeta(k)$, where

$$
\Pi_{1}=\left[\begin{array}{cc}
-P+Q+\bar{A}^{T} P \bar{A} & * \\
\bar{A}_{1}^{T} P \bar{A} & -Q+\bar{A}_{1}^{T} P A_{1}
\end{array}\right]
$$

When $\Pi_{1}<0$, we obtain that $\Delta V<0$. Therefore, the uncertain discrete-time delay system (7) with $w(k)=0$ is stable if $\Pi_{1}<0$.

Let us now prove passivity. From (13), we can easily obtain the following:

$$
\Delta V-2 z^{T}(k) w(k)=\xi^{T} \Pi_{2} \xi
$$

where

$$
\Pi_{2}=\left[\begin{array}{ccc}
-P+Q+\bar{A}^{T} P \bar{A} & * & * \\
\bar{A}_{1}^{T} P \bar{A} & -Q+\bar{A}_{1}^{T} P \bar{A}_{1} & * \\
C+D^{T} P \bar{A} & D^{T} P \bar{A}_{1} & -E^{T}+D^{T} P D
\end{array}\right]
$$

Based on Definition 1, if $\Pi_{2}<0$, we have $\Delta V-2 z^{T}(k) w(k)<0$. That is $\Delta V<2 z^{T}(k) w(k)$. We can choose $V_{1}(x(k))=\frac{1}{2} V(x(k))$ that satisfies Definition 1 , so the system (7) is strictly passive.

Using the Schur complement lemma to $\Pi_{2}$, we obtain the following inequality:

$$
\left[\begin{array}{cccc}
-P+Q & * & * & * \\
0 & -Q & * & * \\
C & 0 & -E^{T} & * \\
\bar{A} & \bar{A}_{1} & D & -P^{-1}
\end{array}\right]<0
$$

Due to $\bar{A}=A+\Delta A+B F K+\triangle B F K, \bar{A}_{1}=A_{1}+\Delta A_{1}$, we obtain the following 


$$
\begin{aligned}
& {\left[\begin{array}{cccc}
-P+Q & 0 & C^{T} & (A+B F K)^{T} \\
0 & -Q & 0 & A_{1}^{T} \\
C & 0 & -E^{T} & D^{T} \\
A+K F B & A_{1} & D & -P^{-1}
\end{array}\right]+\left[\begin{array}{cccc}
0 & 0 & 0 & (\Delta A+\Delta B F K)^{T} \\
0 & 0 & 0 & \Delta A_{1}^{T} \\
0 & 0 & 0 & 0 \\
\Delta A+\Delta B F K & \Delta A_{1} & 0 & 0
\end{array}\right]} \\
& =\left[\begin{array}{cccc}
-P+Q & 0 & C^{T} & (A+B F K)^{T} \\
0 & -Q & 0 & A_{1}^{T} \\
C & 0 & -E^{T} & D^{T} \\
A+K F B & A_{1} & D & -P^{-1}
\end{array}\right]+\left[\begin{array}{cc}
0 & 0 \\
0 & 0 \\
0 & 0 \\
H & 0
\end{array}\right] G(k)\left[\begin{array}{cccc}
M_{0}+M_{2} K & M_{1} & 0 & 0 \\
0 & 0 & 0 & 0
\end{array}\right] \\
& +\left[\begin{array}{cc}
\left(\mathrm{M}_{0}+\mathrm{M}_{2} \mathrm{~K}\right)^{T} & 0 \\
\mathrm{M}_{1}^{T} & 0 \\
0 & 0 \\
0 & 0
\end{array}\right] \mathrm{G}^{T}(\mathrm{k})\left[\begin{array}{cccc}
0 & 0 & 0 & H^{T} \\
0 & 0 & 0 & 0
\end{array}\right]<0
\end{aligned}
$$

By using Lemma1 and Schur complement lemma, we obtain

$$
\left[\begin{array}{ccccccc}
-P & * & * & * & * & * & * \\
0 & -Q & * & * & * & * & * \\
C & 0 & -E^{T} & * & * & * & * \\
A+B F K & A_{1} & D & -P^{-1}+\varepsilon H H^{T} & * & * & * \\
M_{0} & M_{1} & 0 & 0 & -\varepsilon I & * & * \\
0 & 0 & 0 & 0 & 0 & -\varepsilon I & * \\
I & 0 & 0 & 0 & 0 & 0 & -Q^{-1}
\end{array}\right]<0
$$

By left-and-right multiplied by $\operatorname{diag}\left\{P^{-1}, Q^{-1}, I, I, I, I, I\right\}$, inequality (16) can be transformed into the following

$$
\left[\begin{array}{ccccccc}
-P^{-1} & * & * & * & * & * & * \\
0 & -Q^{-1} & * & * & * & * & * \\
C P^{-1} & 0 & -E^{T} & * & * & * & * \\
A P^{-1}+B F K P^{-1} & A_{1} Q^{-1} & D & -P^{-1}+\varepsilon H H^{T} & * & * & * \\
M_{0} P^{-1}+M_{2} K P^{-1} & M_{1} Q^{-1} & 0 & 0 & -\varepsilon I & * & * \\
0 & 0 & 0 & 0 & 0 & -\varepsilon I & * \\
P^{-1} & 0 & 0 & 0 & 0 & 0 & -Q^{-1}
\end{array}\right]<0
$$

Let $Z=P^{-1}, X=Q^{-1}, K_{l}=K Z$, inequality (17) is equal to inequality (10). By the Definition 1, the proof of Theorem 1 is completed.

Furthermore, when $F$ is unknown but satisfies (3) - (6), we have the next theorem.

Theorem 2: Suppose that Assumption 1 holds. If there exist positive definite symmetric matrices $Z, X$, matrix $K_{m}$, a diagonal $R>0$, and $\varepsilon>0$ satisfying the following

$$
\left[\begin{array}{cccccccc}
-Z & * & * & * & * & * & * & * \\
0 & -X & * & * & * & * & * & * \\
C Z & 0 & -E^{T} & * & * & * & * & * \\
A Z+B F_{0} K_{m} & A_{1} X & D & -Z+\varepsilon H H^{T}+B R B^{T} & * & * & * & * \\
M_{0} Z+M_{2} K_{m} & M_{1} X & 0 & 0 & -\varepsilon I & * & * & * \\
0 & 0 & 0 & 0 & 0 & -\varepsilon I & * & * \\
Z & 0 & 0 & 0 & 0 & 0 & -X & * \\
K_{l} & 0 & 0 & 0 & 0 & 0 & 0 & -R \tilde{F}^{-2}
\end{array}\right]<0
$$

then the uncertain discrete-time delay system (7) is robust and strictly passive and the gain matrix $K=K_{m} Z^{-1}$

Proof: Substitute (6) into (10), then we can rewritten (10) as the following

$$
M_{1}=M_{0}+\left[0,0,0, B^{T}, 0,0,0\right]^{T} \Delta\left[K_{m}, 0,0,0,0,0,0,0\right]+\left[K_{m}, 0,0,0,0,0,0\right]^{T} \Delta\left[0,0,0, B^{T}, 0,0,0\right]
$$

where 


$$
M_{0}=\left[\begin{array}{ccccccc}
-Z & * & * & * & * & * & * \\
0 & -X & * & * & * & * & * \\
C Z & 0 & -E^{T} & * & * & * & * \\
A Z+B F_{0} K_{m} & A_{1} X & D & -Z+\varepsilon H H^{T} & * & * & * \\
M_{0} Z+M_{2} K_{m} & M_{1} X & 0 & 0 & -\varepsilon I & * & * \\
0 & 0 & 0 & 0 & 0 & -\varepsilon I & * \\
Z & 0 & 0 & 0 & 0 & 0 & -X
\end{array}\right]
$$

From (6) and the elementary inequality $x^{T} y+y^{T} x \leq \varepsilon x^{T} x+\varepsilon^{-1} y^{T} y$, we have the following $M_{1} \leq M_{0}+\left[0,0,0, B^{T}, 0,0,0\right]^{T} R\left[0,0,0, B^{T}, 0,0,0\right]+\left[K_{m}, 0,0,0,0,0,0\right]^{T} R^{-1} \tilde{F}^{2}\left[K_{m}, 0,0,0,0,0,0\right]$

$$
=\left[\begin{array}{ccccccc}
-Z+K_{m} R^{-1} \tilde{F}^{2} K_{m} & * & * & * & * & * & * \\
0 & -X & * & * & * & * & * \\
C Z & 0 & -E^{T} & * & * & * & * \\
A Z+B F_{0} K_{m} & A_{1} X & D & -Z+\varepsilon H H^{T}+B R B^{T} & * & * & * \\
M_{0} Z+M_{2} K_{m} & M_{1} X & 0 & 0 & -\varepsilon I & * & * \\
0 & 0 & 0 & 0 & 0 & -\varepsilon I & * \\
Z & 0 & 0 & 0 & 0 & 0 & -X
\end{array}\right]
$$

Through Schur complement, we can obtain (18) from (19). Therefore, the system (7) is robust stable and also strictly passive. The proof is completed.

\section{SIMULATION EXAMPLES}

In this section, we give two illustrative examples to verify the effectiveness of main results.

Consider the following uncertain discrete-time system with known actuator failures parameter matrix $F$ and time delay:

$$
\begin{aligned}
& x(k+1)=(A+\Delta A) x(k)+\left(A_{1}+\Delta A_{1}\right) x(k-d)+(B+\Delta B) u(k)+D w(k) \\
& z(k)=C x(k)+E w(k) \\
& x(k)=\varphi(k) \quad k \in[-d, 0]
\end{aligned}
$$

where

$$
\begin{aligned}
& A=\left[\begin{array}{cc}
-0.4 & 0.6 \\
0.6 & 0.4
\end{array}\right], \quad A_{1}=\left[\begin{array}{cc}
0.1 & -0.2 \\
0.4 & 0.2
\end{array}\right], \quad B_{1}=\left[\begin{array}{cc}
0.2 & 0.2 \\
0.1 & 0.5
\end{array}\right], \\
& C=\left[\begin{array}{cc}
-0.1 & 0.1 \\
0.1 & 0.1
\end{array}\right], \quad D=\left[\begin{array}{cc}
0.3 & 0 \\
0 & 0.3
\end{array}\right], \quad \mathrm{E}_{1}=\left[\begin{array}{cc}
0.2 & 0 \\
0 & 0.2
\end{array}\right], \\
& H=\left[\begin{array}{cc}
0.16 & 0.2 \\
0.3 & 0.4
\end{array}\right], \quad M_{0}=\left[\begin{array}{cc}
0.2 & 0.36 \\
0.2 & 0.4
\end{array}\right], \quad \mathrm{M}_{1}=\left[\begin{array}{cc}
0.3 & 0.35 \\
0.25 & 0.4
\end{array}\right], \\
& M_{2}=\left[\begin{array}{cc}
0.26 & 0.15 \\
0.4 & 0.36
\end{array}\right], G(k)=\left[\begin{array}{cc}
\sin (k) & 0 \\
0 & \cos (k)
\end{array}\right], F=\left[\begin{array}{cc}
0.8 & 0 \\
0 & 0.8
\end{array}\right] .
\end{aligned}
$$

Initial state:

$$
x(1)=\left[\begin{array}{l}
1 \\
1
\end{array}\right], x(2)=\left[\begin{array}{l}
1 \\
1
\end{array}\right], w(k)=0.005\left[\begin{array}{c}
\sin (k) \\
\cos (k)
\end{array}\right], \varepsilon=1
$$

\section{Example 1:}

$$
\text { When } \begin{aligned}
F & =\left[\begin{array}{cc}
0.8 & 0 \\
0 & 0.8
\end{array}\right] \text {, solve the LMI in Theorem 1, we obtain } \\
X & =\left[\begin{array}{cc}
2.3006 & -0.8522 \\
-0.8522 & 1.7772
\end{array}\right] \quad Q=X^{-1}=\left[\begin{array}{ll}
0.5285 & 0.2535 \\
0.2535 & 0.6842
\end{array}\right] \quad Z=\left[\begin{array}{ll}
0.7507 & 0.1396 \\
0.1396 & 0.8544
\end{array}\right] \\
P & =Z^{-1}=\left[\begin{array}{cc}
1.3739 & -0.2245 \\
-0.2245 & 1.2072
\end{array}\right] \quad K=K_{l} Z^{-1}=\left[\begin{array}{cc}
0.6333 & -1.5905 \\
-1.6014 & -0.4894
\end{array}\right], \quad K_{l}=\left[\begin{array}{cc}
0.2534 & -0.12705 \\
-1.2705 & -0.6417
\end{array}\right]
\end{aligned}
$$

In this example, our aim is to design a controller, such that the resulting closed-loop system is robust stable and strictly passive. By using LMI in Matlab tool box, we obtain following controlled input and output of the loop-closed systems showed in the Figure.1 and Figure.2. We can verify that the state of the loop-closed system is bounded, converges to zero and strictly passive. 


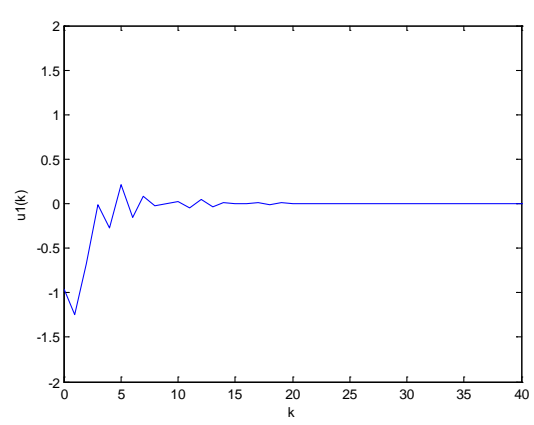

Fig1: Controller input $u(k)$ when $F$ is known

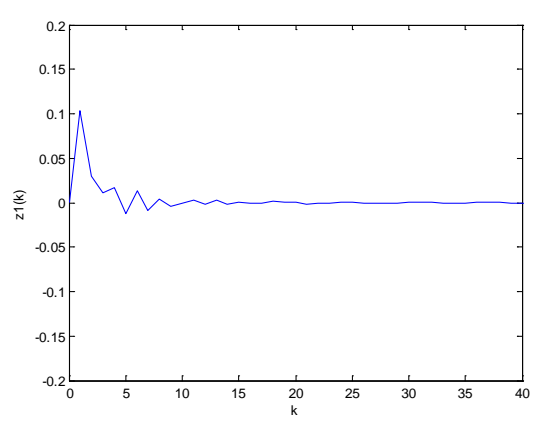

Fi2: Controller output $z(k)$ when $F$ is known

\section{Example 2:}

When $F$ is unknown but satisfies (3)-(6), the sensor fault matrix $F$ is assumed to satisfy $0.1 \leq F \leq 0.9$, solve the LMI in Theorem 2, we obtain

$$
\begin{aligned}
& X=\left[\begin{array}{cc}
2.2672 & -1.2059 \\
-1.2059 & 3.4828
\end{array}\right], \quad Z=\left[\begin{array}{cc}
1.0670 & -0.1031 \\
-0.1031 & 1.1040
\end{array}\right], \quad K_{m}=\left[\begin{array}{cc}
-0.3895 & -0.5691 \\
-0.5691 & -1.1441
\end{array}\right], \\
& Q=X^{-1}=\left[\begin{array}{ll}
0.5406 & 0.1872 \\
0.1872 & 0.3519
\end{array}\right], \quad P=Z^{-1}=\left[\begin{array}{ll}
0.9457 & 0.0883 \\
0.0883 & 0.9141
\end{array}\right], \quad K=K_{m} Z^{-1}=\left[\begin{array}{ll}
-0.4186 & -0.5546 \\
-0.6393 & -1.0961
\end{array}\right]
\end{aligned}
$$

By using LMI in Matlab tool box, we obtain following controlled input and output of the loop-closed systems showed in the Fig.3 and Fig.4. We can verify that the control output of the loop-closed system is bounded, converges to zero and strictly passive.

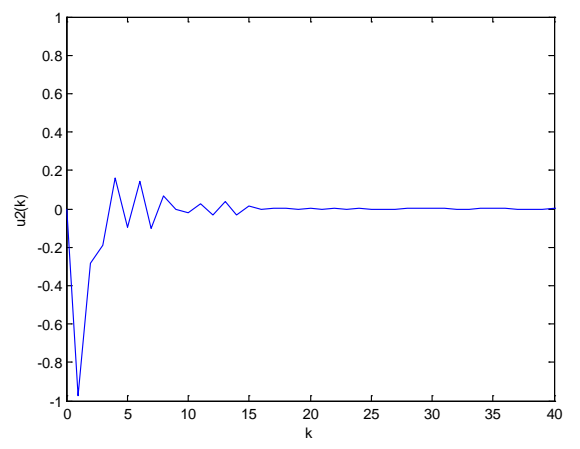

Fig3: Controller input $u(k)$ when $F$ is unknown

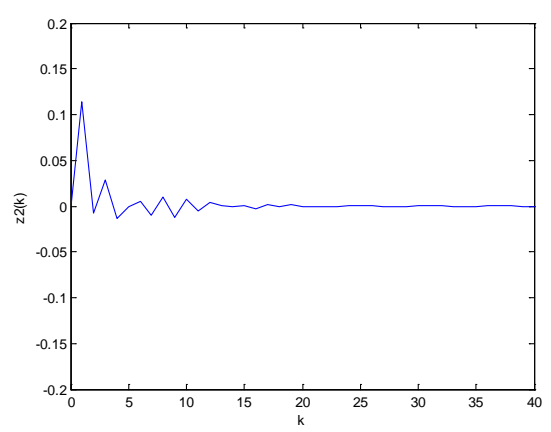

Fig4: Controller output $z(k)$ when $F$ is unkown

\section{CONCLUSION}

In this paper, we study the robust reliable passive control problem against actuator failures for a class of uncertain discrete-time system with time delay using passive control methods. Sufficient conditions for the existence of the robust passive controller have been presented in terms of LMI. Two numerical examples have been given to illustrate the effectiveness of the proposed method.

\section{REFERENCES}

[1]Ai Ling, Xinzhuang Dong, Hui Yang. Passive Control for Uncertain Discrete Time-Delay Singular Systems. Intelligent Networks and Intelligent Systems (ICINIS), 2010 3rd International Conference on. IEEE, 2010: 156-159.

[2] Yanjiang Li, Guangren Duan. Delay-Dependent Robust Passive Control for Uncertain Discrete T-S Fuzzy Time-Delay Systems. Intelligent Computation Technology and Automation (ICICTA), vol 1, 392-395, 2011.

[3] ZhengGuang Wu, Peng Shi, Hongye Su, and Jian Chu. Networked-based robust passive control for fuzzy systems with randomly occurring uncertainties Fuzzy Systems, IEEE Transaction on, Volume 21, 996-971, 2013. 
[4] Feng Liu, Zhihong Guan, Guangxi Zhu. Passive control of chaos in TCP/AQM networks with time delay. Control Conference (CCC), 2010 29th Chinese, IEEE, 2010: 3453-3457

[5] Willems J C. Dissipative Dynamical Systems-Part II: Linear Systems with Quadratic Supply Rates. Archive for Rational Mechanics and Analysis, 45(5): 352-293,1972.

[6] Zhiguang Feng, J Lam, Robust reliable dissipative filtering for discrete delay singular systems. Signal Process, 92(12): 3010-3025, 2012.

[7] Jie Lian, Peng Shi, Zhi Feng. Passivity and passification for a class of uncertain stochastic switched time-delay systems, IEEE Transactions on, 43(1): 3-13, 2013.

[8] Jun Song, Shuping He. Finite-time robust optimal passive control for a class of uncertain nonlinear systems, Control and Decision Conference (CCDC), 221-225, 2013.

[9] Lianqing Su, Xiaodan Zhu, Jiqing Qiu. Robust passive fault tolerant control for uncertain non-linear stochastic systems with distributed delays. Control Conference (CCC), 2010 29th Chinese. IEEE, 2010: 1949-1953.

[10] Ying Yang, YanJun Wang, et al. Robust passive fault-tolerant control for uncertain singular systems. Machine Learning and Cybernetics(ICMLC), 2011 Internationa Conference on. IEEE, 3: 1308-1312, 2011.

[11] R Sakthivel, M Rathika, S Srimanta,etal. Dissipative reliable controller design for uncertain systems and its application. Applied Mathematics and Computation, 263: 107-121, 2015.

[12]Huimei Jia, Zhengrong Xiang, Hamid Reza Karimi. Robust reliable passive control of uncertain stochastic switched time-delay systems. Applied Mathematics and Computation 231: 254-267. 2014.

[13] R Oubellil, M Boukhnifer. Passive fault tolerant control design of energy management system for electric vehicle. Industrial Electronics (ISIE), 2014 IEEE 23rd International Symposium on. IEEE, 2014: 1402-1408.

[14] Yani Souami, Nazith Mechbal, Stephane Ecoutin. Robust Passive Fault Tolerant Control Applied to a Fuel Metering Valve of a Jet Engine. Aerospace Conference, 2015 IEEE. IEEE, 2015: $1-12$.

[15] Z.D. Wang , G.L. Wei, G. Feng. Reliable control for discrete-time piecewise linear systems with infinite distributed delays. Automatica 45(12), 2991-2994, 2009.

[16] J.M Wei, Q Feng, R Ma. Stability analysis robust passive control for uncertain discrete-time singular systems. Procedding of the 2011 International Conference on Advanced Mechatronic Systems, Zhengzhou, China, August 11-13, 2011.

[17] Mahmoud M S. Robust control of discrete systems with uncertain parameters and unknown delay, IEEE Trans Automatica, 36(4): 627-635, 2000. 